\title{
Primer registro para Chile de las diatomeas marinas Nitzschia amabilis, Nitzschia elegantula y Chaetoceros muelleri var. subsalsum
}

First report for Chile of the marine diatoms Nitzschia amabilis, Nitzschia elegantula and Chaetoceros muelleri var. subsalsum

\section{Patricio Rivera $^{1}$ y Fabiola Cruces ${ }^{1}$}

\begin{abstract}
${ }^{1}$ Departamento de Botánica, Facultad de Ciencias Naturales y Oceanográficas, Universidad de Concepción, Casilla 160-C, Concepción, Chile.privera@udec.cl
\end{abstract}

\begin{abstract}
The marine small diatoms Nitzschia amabilis, Nitzschia elegantula and Chaetoceros muelleri var. subsal sum are reported for the first time from Chilean coastal waters. A short diagnosis is included for each taxon, and photomicrographs provide information about the main morphological characteristics.
\end{abstract}

Key words: Morphology, taxonomy, distribution, new records

\section{INTRODUCCIÓN}

Durante el desarrollo de los Proyectos FONDEF DO71-1063 'Manejo biotecnológico de microalgas nativas para la obtención de biodiesel' y FONDEF DO71-1017 'Biotecnología aplicada para la producción de alimentos y dietas balanceadas con la finalidad de mejorar el cultivo del Ostión del Norte’ (Facultad de Ciencias Naturales y Oceanográficas, Universidad de Concepción), se recolectaron muestras de microalgas en distintas localidades de la costa chilena. El análisis de cepas originadas a partir de dichas muestras permitió reconocer por primera vez para estas aguas a las diatomeas Nitzschia amabilis Suzuki, Nitzschia elegantula Grunow y Chaetoceros muelleri Lemmermann var. subsalsum (Lemmermann) Johansen \& Rushforth. Con respecto a esta última especie, ya era conocida para el país la cita de la espora de resistencia de su variedad nominal. En el presente trabajo se entrega una diagnosis de cada uno de estos taxones, junto con fotomicrografías obtenidas de técnicas de microscopía fotónica, electrónica de barrido y de transmisión, para ilustrar sus principales características morfológicas.

\section{Material Y MÉTODOS}

Chaetoceros muelleri var. subsalsum fue encontrado en material de fitoplancton costero recolectado en diciembre de 2005 en Bahía de Coliumo (36³2’S, 7257’W); Nitzschia amabilis y Nitzschia elegantula fueron obtenidas del contenido estomacal del Ostión del Norte, Argopecten purpuratus Lamarck, recolectado en Bahía Salada, Caldera
( $27^{\circ} 38^{\prime} \mathrm{S}, 71^{\circ} 00^{\prime} \mathrm{W}$ ) en octubre de 2008 y en marzo de 2009 respectivamente. Células aisladas desde estas muestras fueron cultivadas en medio Walne + Si (Walne 1970) en el Laboratorio de Microalgas de la Universidad de Concepción a una temperatura de $15 \pm 2^{\circ} \mathrm{C}$, con un fotoperíodo de $16 \mathrm{~h}$ luz y $8 \mathrm{~h}$ de oscuridad y a 10-20 $\mu$ mol de fotones por metro cuadrado por segundo. El material estudiado se encuentra depositado en la Colección Diatomológica de la Universidad de Concepción, Chile (DIAT-CONC). Luego de la eliminación de la materia orgánica según el método de Hasle \& Fryxell (1970), el material fue analizado con microscopía fotónica (MO, Zeiss Fotomicroscopio III) y con microscopía electrónica de barrido (MEB, Jeol JSM-6380LVY) y de transmisión (MET, Jeol 1200 EX II). La terminología usada corresponde a la sugerida por Anonymous (1975) y Ross et al. (1979).

\section{Resultados y Discusión}

Nitzschia amabilis Suzuki in Suzuki et al. (2010) (Fig. $1 \mathrm{~A}-\mathrm{K})$

Descripción original: Suzuki et al. (2010), p. 223, fig. 1.

Sinonimia: Nitzschia laevis Hustedt, p. 662, figs. 116-118 (1939), non Frenguelli, p. 91, lám. VIII, fig. 3 (1923); Simonsen, p. 261, lám. 385, figs. 10-18 (1987). Krammer \& Lange-Bertalot, p. 72, fig. 65. 1-2 A (1988). Witkowski et al., p. 387, lám. 190, figs. 1-6 (2000). 


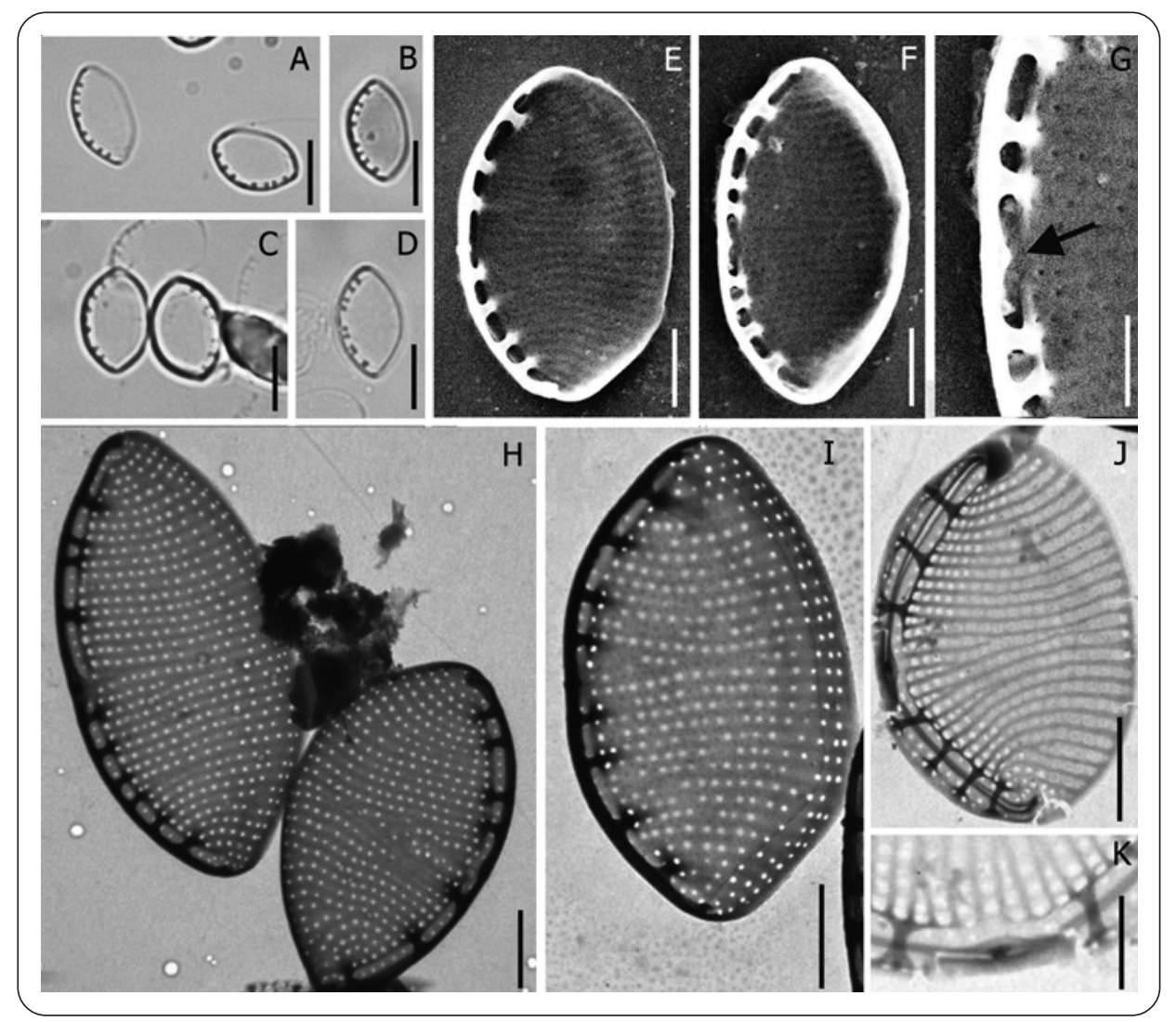

Figura 1. Nitzschia amabilis. A-D) MO; E-G) MEB; H-K) MET. A-D) Valvas anchamente elípticas. E-F) Fíbulas centrales más distanciadas entre sí. G) Pequeña protuberancia marginal del canal del rafe (flecha). H-I) Estrías uniseriadas; área hialina irregular al centro de la valva. J) Canal del rafe. K) Rafe interrumpido al centro. Escalas: A$\mathrm{D}=\mathbf{1 0} \mu \mathrm{m} ; \mathrm{E}-\mathrm{F}, \mathrm{H}-\mathrm{J}=\mathbf{2} \mu \mathrm{m} ; \mathrm{G}, \mathrm{K}=\mathbf{1}$ $\mu \mathrm{m} /$ Nitzschia amabilis. A-D) MO; E-G) MEB; H-K) MET. A-D) Broadly elliptic valves. E-F) The two median fibulae distant. G) Small protuberance of marginal canal raphe system (arrow). H-I) Uniseriate striae; central hyaline area irregular in shape. J) Canal raphe system. K) Central nodule. Scale bars: $A-D=10$ $\mu \mathrm{m} ; \mathrm{E}-\mathrm{F}, \mathrm{H}-\mathrm{J}=2 \mu \mathrm{m} ; \mathrm{G}, \mathrm{K}=1 \mu \mathrm{m}$

Material estudiado: Bahía Salada, Caldera, DIAT-CONC M-3349, 3353.

Distribución: especie marina, cosmopolita (Witkowski et al. 2000). No ha sido señalada anteriormente para Chile.

Valvas anchamente elípticas con los extremos obtusos redondeados, levemente sobresalientes (Fig. 1 A-F, H-J). Longitud 8-12 $\mu \mathrm{m}$, ancho 5-6 $\mu \mathrm{m}$. Rafe lateral, interrumpido en el medio de la valva (Fig. $1 \mathrm{~J}-\mathrm{K}$ ); fíbulas anchas y relativamente largas, $10-14$ en $10 \mu \mathrm{m}$, unidas usualmente a dos interestrías (Fig. $1 \mathrm{H}$ ), a veces a tres (Fig. $1 \mathrm{I}-\mathrm{J}$ ), las dos fíbulas centrales están más separadas entre sí formando un interespacio central (Fig. 1 E, I-J). Estrías transapicales uniseriadas (Fig. $1 \mathrm{H}-\mathrm{J}$ ), difíciles de observar en el microscopio fotónico, 32-34 en $10 \mu \mathrm{m}$, a veces interrumpidas en la parte central de la valva dejando espacios hialinos de forma irregular (Fig. 1 H-I).

Las características del material chileno concuerdan con aquellas presentes en $N$. amabilis, aunque el contorno valvar de esta especie es más linear-lanceolado. Sin embargo el largo de las valvas del material chileno (8-12 $\mu \mathrm{m})$ es un contínuo con los 12-25 $\mu \mathrm{m}$ de la $N$. laevis Hustedt, y el ancho es similar, existiendo por lo tanto solo una diferencia en la relación largo/ancho. El número de estrías presentes en nuestro material (32 a 34 en $10 \mu \mathrm{m}$ ) es levemente menor que en el material tipo (37 en $10 \mu \mathrm{m}$ según Simonsen 1987, p. 261) y que en las células estudiadas por Witkowski et al. (2000, 40 en $10 \mu \mathrm{m}$ ), pero según Krammer \& Lange-Bertalot (1988) la cantidad de estrías varía entre 32 a 36 y pueden llegar hasta 50 en $10 \mu \mathrm{m}$. Una característica no claramente visible con microscopía fotónica (Fig. 1 C-D) se relaciona con la mayor separación que existe entre las dos fíbulas centrales (Fig. 1 E, I-J). Con microscopía electrónica se observa la presencia de una pequeña protuberancia en el margen del canal del rafe, ubicada en el centro de la valva (Fig. 1 F-H), la cual, con bajos aumentos, puede ser confundida con una fíbula.

Nitzschia elegantula Grunow in Van Heurck (1881) (FIG. 2 A-B)

Descripción original y otras: Van Heurck (1881), lám. 69, fig. 22a; Krammer \& Lange-Bertalot (1988), p. 120, lám. 83, figs. 20-24, 25-26?; Witkowski et al. (2000), p. 379, lám. 207, figs. 10-13. 

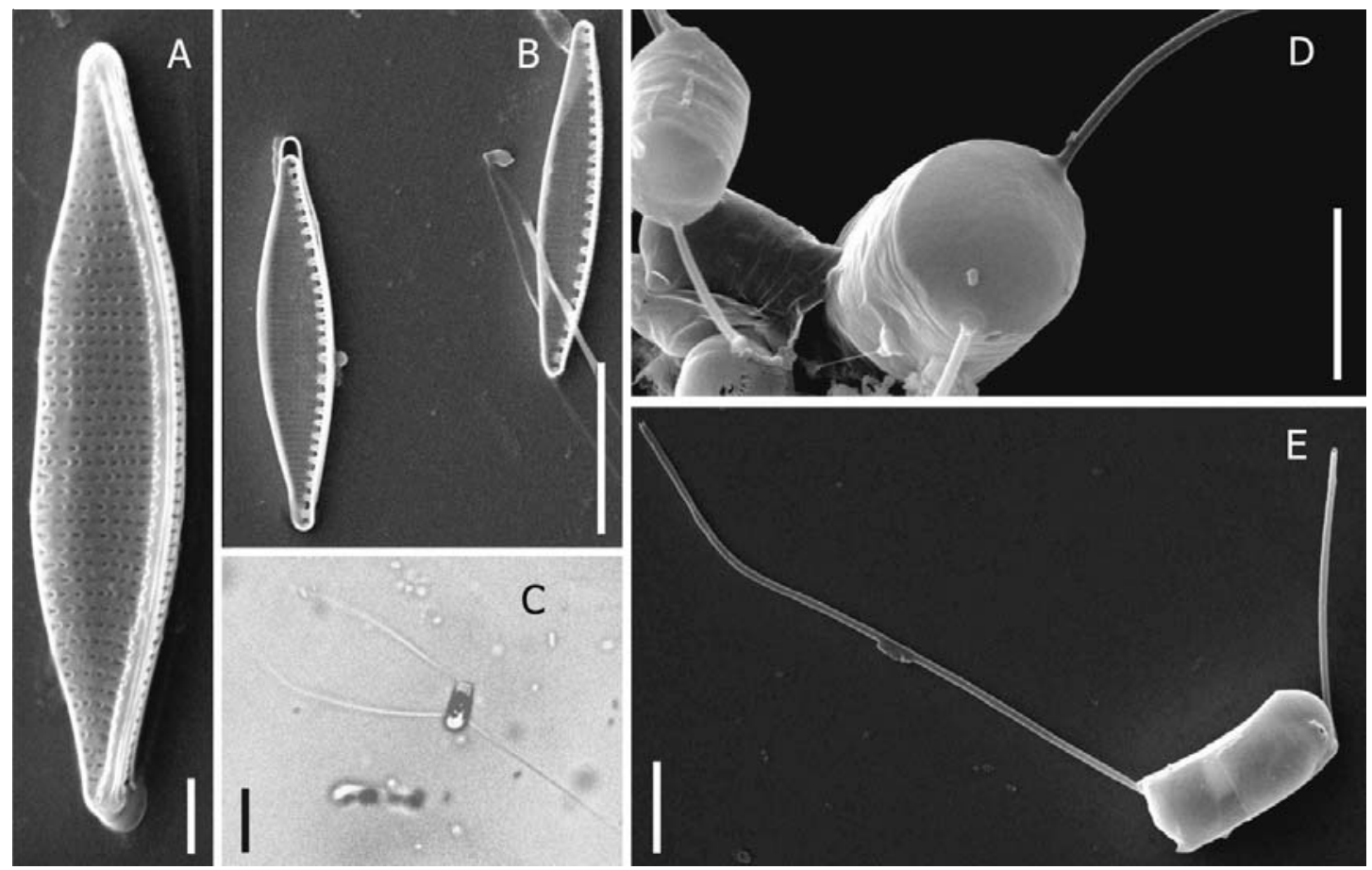

Figura 2. A-B, D-E) MEB; C) MO. A-B. Nitzschia elegantula. A) Valva contraída en el centro; estrías uniseriadas. B) Fíbulas igualmente espaciadas; valvas lanceoladas no contraídas en el centro. C-E) Chaetoceros muelleri var. subsalsum. C) Setas emergen de los ápices de la valva en un ángulo inferior a 45․ D) Valvas elípticas a subcirculares. E) Setas delgadas, largas; valvas convexas. Escalas: $A=2 \mu \mathrm{m} ; \mathrm{B}-\mathrm{C}=10$ $\boldsymbol{\mu m}$; D-E = $\mathbf{5} \boldsymbol{\mu \mathrm { m }}$ / A-B, D-E) MEB; C) MO. A-B) Nitzschia elegantula. A) Valve with constricted margin; striae uniseriate. B) Fibulae evenly spaced; lanceolate valves with not constricted margins. C-E) Chaetoceros muelleri var. subsalsum. C) Setae emerging from the poles of valve in an angle of less than 45 o to apical axis. D) Elliptic to subcircular valves. E) Setae thin, long; convex valves. Scale bars: $A=2 \mu \mathrm{m} ; B-C=10 \mu \mathrm{m} ; \mathrm{D}-\mathrm{E}=5 \mu \mathrm{m}$

Material estudiado: Bahía Salada, Caldera, DIAT-CONC M-3323, 3327.

Distribución: de acuerdo con Witkowski et al. (2000) es una especie cosmopolita propia de ambientes marinos costeros y aguas interiores con alto contenido de electrolitos. No había sido anteriormente señalada para Chile.

Células reunidas por mucílago en conjuntos de forma irregular. Frústulos rectangulares en vista conectival. Valvas lanceoladas a linear-lanceoladas con los márgenes algo contraídos en la parte media (Fig. 2 A), y con extremos diferenciados, rostrados a subcapitados (Fig. 2 A-B). Largo 15-24 $\mu \mathrm{m}$, ancho 3-4,5 $\mu \mathrm{m}$. Rafe lateral, no interrumpido en la parte media; fíbulas anchas, notorias, igualmente espaciadas, 9-14 en $10 \mu \mathrm{m}$. Estrías transapicales uniseriadas, 23-26 en $10 \mu \mathrm{m}$.

El grado de constricción de la valva varía en los diferentes individuos y en algunos parece faltar (Fig. 2 B). Esta característica fue también observada por Krammer \& LangeBertalot (1988, lám. 83, figs. 25-26).
ChaEtoceros muelleri LemMERMANN VAR. SUBSALSUM (LEMMERMANN) JOHANSEN \& RUSHFORTH (1985) (FIG. 2 C-E)

Descripción original: Johansen \& Rushforth (1985), p. 445, figs. 1-60.

Basiónimo: Chaetoceros subsalsum Lemmermann, (1904), p. 141, lám. 1, fig. 16.

Material estudiado: Bahía de Coliumo, DIAT-CONC M3330.

Distribución: tanto la variedad nominal como la variedad subsalsum están generalmente presentes en aguas marinas salobres y en lagos con alta conductividad (Johansen \& Rushforth 1985, Rines \& Hargraves 1988). No ha sido señalada anteriormente para Chile. Esporas de resistencia de la variedad nominal fueron encontradas por Rebolledo et al. (2005) en sedimentos provenientes de fiordos del sur de Chile (Canal Puyuhuapi). 
Frústulos solitarios, rectangulares en vista conectival, con un cloroplasto. Eje pervalvar 7-12 $\mu \mathrm{m}$, eje apical 3-7 $\mu \mathrm{m}$. Valvas elípticas a subcirculares, planas o convexas, sin procesos labiados (Fig. 2 D). Setas largas y delgadas, rectas o algo curvadas hacia el extremo, emergiendo desde los ápices de las valvas y proyectándose hacia el exterior en un ángulo agudo menor a 45 grados (Fig. 2 C, E).

La variedad subsalsum se diferencia de la variedad nominal por carecer de procesos labiados sobre la superficie de las valvas. Además, Johansen \& Rushforth (1985) observaron en esta variedad que las setas están ornamentadas con poroides de contorno rectangular, dispuestos en líneas espiraladas. En el presente trabajo, esta característica puede ser visualizada en forma parcial al aumentar significativamente el tamaño de la Figura 2 D. En el material analizado sólo se observaron células aisladas, pero según la literatura esta variedad también se puede encontrar formando cortas cadenas y sus esporas de resistencia presentan la pared externa usualmente lisa.

El hallazgo por primera vez en Chile de los taxones incluídos en el presente trabajo, evidenciaría que otras especies, de pequeño tamaño, pueden estar pasando desapercibidas en los análisis rutinarios de muestras de microalgas. Este ha sido el caso, entre otros, de lo ocurrido con las diatomeas Minidiscus chilensis Rivera (Rivera \& Koch 1984) y Extubocellulus spinifer (Hargraves \& Guillard) Hasle, von Stosch \& Syvertsen (Rivera et al. 2010), que siendo abundantes en la costa chilena sólo fueron descubiertas mediante el uso de técnicas de microscopía adecuadas (microscopía electrónica de barrido, de transmisión, secado del material mediante punto crítico).

\section{Agradecimientos}

Agradecemos a dos evaluadores anónimos por su aporte en la revisión crítica del texto, y al personal del Laboratorio de Microscopía Electrónica (Universidad de Concepción) por su apoyo con los equipos. Estudio financiado por los Proyectos FONDEF DO71-1063 'Manejo biotecnológico de microalgas oleaginosas nativas para la obtención de biodiesel’ y FONDEF DO71-1017 ‘Biotecnología aplicada a producción de alimentos y dietas balanceadas para optimizar el cultivo del Ostión del Norte’.

\section{LiterATURA CITADA}

Anonymous. 1975. Proposal for a standardization of diatom terminology and diagnosis. Nova Hedwigia Beiheft 53: 323-354.
Frenguelli J. 1923. Diatomeas Argentinas. I. Contribuciones para la sinopsis de las diatomeas Argentinas. Diatomeas del Río Primero en la ciudad de Córdoba. Boletín de la Academia Nacional de Ciencias en Córdoba (República Argentina) 18: 13-119.

Hasle GR \& G Fryxell. 1970. Diatoms: cleaning and mounting for light and electron microscopy. Transaction of the American Microscopical Society 89: 469-474.

Hustedt F. 1939. Die diatomeenflora des Küstengebietes der Norsee vom Dollart bis zur Elbemündung. I. Die Diatomeenflora in den Sedimenten der unteren Ems sowie auf den Watten in der Leybucht, des Memmert und bei der Insel Just. Adhandlungen Naturwissenschaftliger Verein zu Bremen 31(3): 571-677.

Johansen J \& S Rushforth. 1985. A contribution to the taxonomy of Chaetoceros muelleri Lemmermann (Bacillariophyceae) and related taxa. Phycologia 24(4): 437-447.

Krammer K \& H Lange-Bertalot. 1988. Bacillariophyceae. 2: Bacillariaceae, Epithemiaceae, Surirellaceae. En: Ettl H, J Gerloff, H Heynig \& D Mollehauer (eds). Süsswasserflora von Mitteleuropa 2/2: 1-596. Fisher, Stuttgart.

Lemmermann E. 1904. Das plankton schwedischer Gewässer. Arkiv für Botanik 2: 1-209.

Rebolledo L, C Lange, D Figueroa, S Pantoja, P Muñoz \& R Castro. 2005. $20^{\text {th }}$ century fluctuations in the abundance of siliceous microorganisms preserved in the sediments of the Puyuhuapi Channel ( $\left.44^{\circ} \mathrm{S}\right)$, Chile. Revista Chilena de Historia Natural 78(3): 469-487.

Rines JEB \& PE Hargraves. 1988. The Chaetoceros Ehrenberg (Bacillariophyceae) Flora of Narragansett Bay, Rhode Island, U.S.A. Bibliotheca Phycologica 79: 1-196.

Rivera P \& P Koch. 1984. Contributions to the diatom flora of Chile. II. En: Mann D (ed). Proceedings of the Seventh International Diatom Symposium Philadelphia, pp. 279298, Koeltz Science Publishers, Koenigstein.

Rivera P, F Cruces \& I Inostroza. 2010. Extubocellulus spinifer (Hargraves \& Guillard) Hasle, Von Stosch \& Syvertsen (Cymatosiraceae, Bacillariophyceae): First report of the species from the Southeastern Pacific with comments on the variability of some of its morphological features. Gayana Botánica 67(2): 153-157.

Ross R, EJ Cox, NI Karayeva, DG Mann, TBB Paddox, R Simonsen \& PA Sims. 1979. An amended terminology for the siliceous components of the diatom cell. Nova Hedwigia Beiheft 64: 513-533.

Simonsen R. 1987. Atlas and catalogue of the diatom types of Friedrich Hustedt, Vol. 1, Catalogue 525 pp., Vol. 2 Atlas Pls. 1-395, Vol. 3 Pls. 396-772. J. Cramer, Berlin / Stuttgart. 
Suzuki H, T Nagumo \& J Tanaka. 2010. Nitzschia amabilis nom. nov., a new name for the marine species $N$. laevis Hustedt. Diatom Research 25(1): 223-224.

Van Heurck H. 1881. Synopsis des diatomées de Belgique, Pls. 31-77. Ducaju et Cie., Anvers.

Walne RR. 1970. Studies on food value of nineteen genera of algae to juvenile bivalves of the genera Ostrea,
Crassostrea, Mercenaria and Mytilus. Fishery Investigations Ministry of Agriculture, Fisheries and Food, Series II 26: 1-45.

Witkowski A, H Lange-Bertalot \& D Metzeltin. 2000. Diatom flora of marine coast I. En: Lange-Bertalot H (ed). Iconographia diatomologica, Annotated diatom micrographs 7: 1-925 pp. Koeltz, Königstein.

Recibido el 23 de agosto de 2010 y aceptado el 17 de diciembre de 2010 